\title{
Association of increased erythrocyte osmotic resistance with haematological and histopathological findings in dogs with a congenital extrahepatic portosystemic shunt
}

\author{
Kristína Řeháková ${ }^{1}$, Ivana Uhríková ${ }^{1}$, Leona Raušerová-Lexmaulová ${ }^{2}$, Jana Lorenzová ${ }^{2}$ \\ Ladislav Stehlík ${ }^{3}$, Eva Jánová ${ }^{4}$, Ondřej Škor ${ }^{5}$, Jaroslav Doubek ${ }^{1}$ \\ ${ }^{1}$ University of Veterinary and Pharmaceutical Sciences Brno, Faculty of Veterinary Medicine, Small Animal \\ Clinical Laboratory, ${ }^{2}$ Small Animal Clinic, Department of Surgery and Orthopaedics, ${ }^{3}$ Department of Diagnostic \\ Imaging, ${ }^{4}$ Department of Animal Genetics, Brno, Czech Republic \\ ${ }^{5}$ Veterinary Clinic Bílá Hora, Prague, Czech Republic
}

Received October 14, 2012

Accepted August 28, 2013

\begin{abstract}
The aim of the study was to investigate changes in erythrocyte osmotic resistance in relation to haematological and histological changes in dogs with a congenital portosystemic shunt. Osmotic fragility tests with complete blood counts and liver histological examinations were performed in 12 dogs with single extrahepatic portosystemic shunt confirmed by surgical exploration. Laboratory results were compared with those from 30 healthy dogs. Dogs with portosystemic shunt had a significantly increased erythrocyte osmotic resistance $(P<0.01)$ with $5 \%, 50 \%$ and $90 \%$ haemolysis corresponding to $0.45 \%, 0.35 \%$ and $0.30 \% \mathrm{NaCl}$ solution, respectively. Statistical analyses revealed no correlation between haematological indicators and the osmotic fragility test results. Increased osmotic resistance was significantly associated with hepatic lipogranulomas. Based on these results, dogs with a congenital portosystemic shunt have a significantly increased erythrocyte osmotic resistance suggesting impaired red blood cell deformability. Osmotic resistance test that until now was not studied in canine hepatopathies seems to be independent of routinely performed haematological tests.
\end{abstract}

Portosystemic vascular anomaly, osmotic fragility, liver biopsy, canine

Portosystemic shunt (PSS) is the most common vascular anomaly in dogs. Persistent communication in single PSS allows blood to bypass the liver and be delivered directly from the portal bloodstream into systemic circulation. All laboratory abnormalities are related to secondary metabolic changes due to progressive liver atrophy (Mathews and Bunch 2005).

In human medicine, chronic liver diseases are associated with increased erythrocyte osmotic resistance (OR) due to accumulation of membrane cholesterol as a result of impaired lipid metabolism. This change in membrane composition with an increased surface area to volume ratio morphologically corresponding to target cells, has a direct effect on red blood cell deformability (Vassiliadis et al. 2010). Target cells are commonly also seen in dogs with chronic liver disorders, but the alterations in erythrocyte OR in hepatopathies have not been described, yet. A similar tendency for increased erythrocyte osmotic resistance in a portosystemic shunt could suggest a similar pathogenesis to impaired erythrocyte deformability in humans with chronic liver disease. Liver biopsy is the gold standard in the diagnostics of hepatic disorders.

The aim of this study was to investigate changes in erythrocyte osmotic resistance in dogs with a single extrahepatic portosystemic shunt, and to determine associations with alterations in the haematology profile and liver histopathological findings. 


\section{Animals \\ Materials and Methods}

Twelve dogs with a history consistent with the diagnosis of portosystemic shunt confirmed by surgical exploration were used in this study. Yorkshire Terrier, accounting for 7 cases, was the most common breed identified in the study. Other breeds included one each of the following: Bichon Frise, Toy Poodle, West Highland White Terrier, Cairn Terrier and a mixed-breed dog. The median age and median body weight of the dogs with a portosystemic shunt was 13 months (range 3 to 28 months) and $2.2 \mathrm{~kg}$ (range 1.5 to $8.5 \mathrm{~kg}$ ), respectively. Seven animals were males, five were females. The control group consisted of 30 privately owned healthy dogs.

\section{Haematological examination}

In all dogs, the peripheral blood for laboratory analysis was collected from the jugular vein. Quantitative haematological examination (Analyser Celltac alpha MEK 6318, Nihon Kohden, Japan) with a blood smear preparation was performed from blood collected to ethylenediaminetetraacetic acid (EDTA) (Tapval, Dispolab, Czech Republic). Haematological examination included red blood cell (RBC) count, haematocrit (Ht) value, haemoglobin $(\mathrm{Hb})$ concentration, mean cell volume $(\mathrm{MCV})$, mean cell haemoglobin $(\mathrm{MCH})$, mean cell haemoglobin concentration (MCHC), white blood cell (WBC) count and platelet count determination. The presence of target cells was proved by microscopic examination of the blood smear.

\section{Osmotic fragility test}

The blood samples for osmotic fragility test (OFT) were collected on lithium-heparin (Tapval, Dispolab, Czech Republic) a suitable anticoagulant for hypotonic red blood cell lysis (Sláma et al. 2006). The OFT was performed according to the method of Dacie and Lewis (Comazzi et al. 2002) by adding $25 \mu 1$ of blood into a $2.5 \mathrm{ml}$ $\mathrm{NaCl}$ solution of decreasing concentrations of $0.90-0.10 \%$. After $30 \mathrm{~min}$ of incubation at room temperature and centrifugation $(165 \mathrm{~g}, 10 \mathrm{~min}$ ), the supernatant absorbance was measured at $540 \mathrm{~nm}$ (ELISA reader ELX 808 BioTek Instruments Inc., USA). Supernatant from saline was used as a negative control without provoking haemolysis; supernatant from $0.1 \% \mathrm{NaCl}$ solution served as positive control with complete haemolysis.

\section{Histological examination}

Liver biopsy samples were obtained from 10 dogs with PSS during surgical shunt attenuation. Samples were routinely stained with haematoxylin-eosin and evaluated using an individual score system for portal area changes and for parenchymal changes (Simpson et al. 1997; Isobe et al. 2008; Lee et al. 2011). In the portal area, absence of portal vein (0-3), arteriolar proliferation (0-3), biliary hyperplasia (0-2) and portal fibrosis (0 or 1$)$ were scored. In the parenchyma, steatosis (0-3), lipogranulomas (0-3), pigment accumulation (0-2), inflammation ( 0 or 1$)$ and necrosis ( 0 or 1$)$ were evaluated.

\section{Statistical analysis}

Haematological results were reported as median values. A cumulative curve of haemolysis was drawn from the mean values for both PSS and control group. The mean concentrations of $\mathrm{NaCl}$ solution corresponding to the minimum (less than 5\% haemolysis), medium (50\% haemolysis) and maximum (more than $90 \%$ haemolysis) osmotic fragility were calculated. Mann-Whitney test was used to compare haematological data, Student's $t$-test for changes in OFT. The level of significance was set at $P<0.05$. Pearson's correlation coefficient was used for correlation analyses between osmotic fragility and the haematocrit, haemoglobin, MCV, MCH and $\mathrm{MCHC}$ values. The association between abnormalities in OR and histological findings in the PSS group was evaluated using general linear modelling (GLM) (Statistica 6.0, Statsoft Inc., USA).

\section{Results}

In haematological examination, significant differences were found for $\mathrm{RBC}$ count, $\mathrm{Ht}$ and $\mathrm{Hb}$ concentrations as well as for $\mathrm{MCV}, \mathrm{MCH}$ and $\mathrm{MCHC}$ values $(P<0.01$, Table 1$)$. Microcytosis was observed in 10 dogs $(n=12)$; decreased $\mathrm{Hb}$ concentration was found only in 3 dogs $(n=12)$. Significant differences were also found for the WBC count. Dogs with a portosystemic shunt and healthy dogs did not differ in the platelet count. Target cells on the blood smear were seen in $50 \%$ of dogs with PSS.

The OFT revealed significantly increased erythrocyte resistance with 5\%, 50\%, and 90\% haemolysis corresponding to the mean values of $0.45 \%, 0.35 \%$ and $0.30 \% \mathrm{NaCl}$ solution, respectively $(P<0.01)$. The cumulative curve of haemolysis was shifted toward the left compared to healthy dogs (Fig. 1). Mean corpuscular fragility (the concentration of $\mathrm{NaCl}$ producing $50 \%$ haemolysis) was decreased in $75 \%$ of dogs. No dogs had increased osmotic fragility. Correlation analyses revealed that none of the haematological indicators were correlated with the results of the osmotic fragility test. 
Table 1. Results of haematological examination in dogs with a portosystemic shunt.

\begin{tabular}{lcccccccc}
\hline Indicator & $\begin{array}{c}\text { Erys } \\
\left(\times 10^{12} / 1\right)\end{array}$ & $\begin{array}{c}\mathrm{Ht} \\
(\mathrm{g} / \mathrm{l})\end{array}$ & $\begin{array}{c}\mathrm{Hb} \\
(\mathrm{g} / \mathrm{l})\end{array}$ & $\begin{array}{c}\mathrm{MCV} \\
(\mathrm{fl})\end{array}$ & $\begin{array}{c}\mathrm{MCH} \\
(\mathrm{pg})\end{array}$ & $\begin{array}{c}\text { MCHC } \\
(\mathrm{g} / \mathrm{l})\end{array}$ & $\begin{array}{c}\text { Leucs } \\
\left(\times 10^{9} / 1\right)\end{array}$ & $\begin{array}{c}\text { Plts } \\
\left(\times 10^{9} / 1\right)\end{array}$ \\
\hline Median & $6.9^{*}$ & $0.41^{*}$ & $129^{*}$ & $59.9^{*}$ & $19.1^{*}$ & $316^{*}$ & $12.3^{*}$ & 225 \\
\hline
\end{tabular}

Erys - erythrocytes, $\mathrm{Ht}$ - haematocrit, $\mathrm{Hb}$ - haemoglobin, $\mathrm{MCV}$ - mean cell volume, $\mathrm{MCH}$ - mean cell haemoglobin, $\mathrm{MCHC}$ - mean cell haemoglobin concentration, Leucs - leukocytes, Plts - platelets, ${ }^{*} P<0.01$

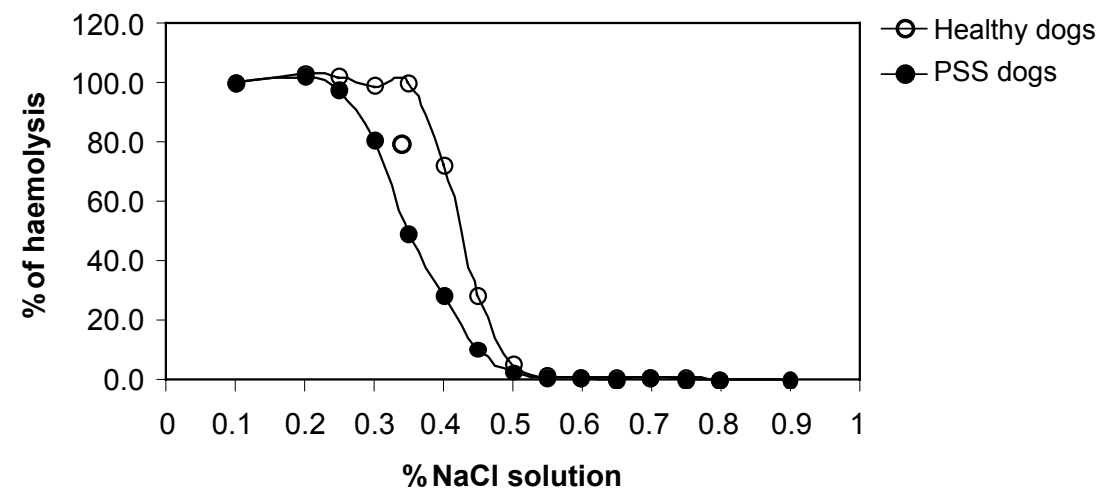

Fig. 1. Cumulative curve of haemolysis in dogs with portosystemic shunt and controls PSS - portosystemic shunt

Table 2. Results of liver histopathological examination in dogs with a portosystemic shunt.

\begin{tabular}{llcccccccccc}
\hline Change & IV & IA & IBD & Steatosis & LG & Pigment & INF & Necrosis & Fibrosis & Total PORS & Total PARS \\
\hline Mean score & 1.8 & 2 & 0.8 & 0.8 & 0.7 & 0.3 & 0.5 & 0.4 & 0.2 & 4.8 & 2.7 \\
\hline
\end{tabular}

IV - absence of interlobular vein, IA - interlobular arteriolar proliferation, IBD - interlobular bile duct hyperplasia, LG - lipogranuloma, INF - inflammation, PORS - portal score, PARS - parenchymal score

Results of the semi-quantitative examination of liver histological changes are presented in Table 2. The most common abnormalities of the portal area (triad) were the absence of the interlobular vein and the arteriolar proliferation that were seen in all animals, followed by biliary hyperplasia observed in 7 of 10 dogs. Portal fibrosis was identified in only two cases. Of the parenchymal changes, lipogranuloma formation was predominant $(6 / 10$ dogs). An inflammatory reaction was observed in half of the dogs $(5 / 10)$, followed by steatosis (4/10), necrosis (4/10) and pigment accumulation $(2 / 10)$.

Haemoglobin concentrations, $\mathrm{MCH}$ and $\mathrm{MCHC}$ values were significantly related to the total parenchymal score. Furthermore, haemoglobin and $\mathrm{MCHC}$ values had simultaneously significant associations with the total portal score. Erythrocyte osmotic resistance was significantly connected only to parenchymal changes. 


\section{Discussion}

From haematological examination, anaemia is considered to be a characteristic finding in PSS. However, its frequency is in fact highly variable, at 25-72\% (Bunch et al. 1995; Simpson et al. 1997). Median Hb concentrations in dogs with PSS are usually lower than in healthy dogs, but may persist in reference intervals. This tendency to the anaemic syndrome was also observed in our study, where median $\mathrm{Hb}$ in dogs with PSS was $129 \mathrm{~g} / \mathrm{l}$. Absence of a more severe decrease in haematocrit can be a result of dehydration due to general mechanisms: decreased water intake, $\mathrm{Pu} / \mathrm{Pd}$, vomiting or diarrhoea.

Regardless of the presence or absence of anaemia, microcytosis seems to be a predominant haematological abnormality. Nevertheless, its origin is still not completely understood. The frequency of microcytosis in our study (in 10 of 12 dogs), was slightly higher than previously published (Bunch et al. 1995; Niles et al. 2001). In general, the major cause of microcytosis in dogs is iron-deficiency anaemia due to chronic blood loss, since accessible iron is limiting for haemoglobin synthesis. However, bleeding tendency is a rare finding of PSS. Moreover, iron-deficiency anaemia is associated with low $\mathrm{MCH}$, $\mathrm{MCHC}$ and low iron storage. MCHC values in our study were decreased in only $40 \%$ of dogs with low MCV. As reported previously, hypochromia in PSS can be an occasional abnormality and iron content in the liver and in the bone marrow is usually increased (Simpson et al. 1997). On the other hand, excessive pigment accumulation can also be absent, as proved in 9 of 10 dogs in our study and as described in previous studies (Bunch et al. 1995). Microcytosis can rarely be also associated with the anaemia of inflammatory disease that is characterized by mild degree, normochromia and functional iron deficit due to transport alterations. Although the WBC count was significantly higher in the PSS group, median values remained within the reference interval, with only two dogs having leukocytosis. At the same time, an inflammatory reaction was seen in one half of liver biopsy samples, underlining that WBC count is not a sensitive marker for parenchymal liver inflammation in PSS. In general, none or only a slight increase in sensitive inflammatory markers (C-reactive protein, ferritin and ceruloplasmin), was previously observed in dogs with portosystemic vascular anomalies (Bunch et al. 1995; Nakamura et al. 2008). This finding indicates that liver inflammatory reaction may not be severe enough to be a major factor in the pathogenesis of the anaemic syndrome in PSS. For 3 dogs that were aged 3-4 months, MCV could have been influenced by a tendency to macrocytosis due to incomplete erythropoiesis.

Until now, no strict relationship to iron deficiency or inflammatory disease has been found, and some authors have formulated the hypothesis that microcytosis is a result of postsynthetic modification rather than primary erythropoiesis dysfunction (Simpson et al. 1997). During chronic liver disease, free serum cholesterol accumulation within the erythrocyte membrane is responsible for increased surface-to-volume ratio making red blood cells more resistant to hypotonic stress. These rigid target cells with decreased membrane fluidity are more easily entrapped by activated macrophages in the spleen sinusoids, leading to overall decreased erythrocyte survival. Iron transfer from plasma transferrin to cells requires internalization of the ferrotransferrin bound to the membrane transferrin receptor. Normal fluidity seems to be an important condition for correct internalization of the receptor-ferrotransferrin complex. Therefore significant changes in erythrocyte membrane composition associated with decreased fluidity may contribute to inadequate iron utilization (Weiss 2009).

Increased erythrocyte OR in dogs with PSS was demonstrated by significantly decreased concentrations of $\mathrm{NaCl}$ solution responsible for 5, 50 and $90 \%$ haemolysis. Changes in OR in dogs with the hepatic disease are not described, and to the authors' knowledge, only one study has focused on changes in osmotic fragility in feline hepatopathies (Elias et al. 
2004). The authors found significantly decreased OR at the level of maximum haemolysis and proposed a hypothesis that increased erythrocyte fragility is mostly due to stress from toxic metabolites released during liver damage rather than due to progressive changes in erythrocyte membrane composition. However, the cats only met general criteria for hepatopathy (high liver enzyme activities, icterus) and no other disease classification was presented. In our study, erythrocyte osmotic resistance was significantly increased compared to healthy dogs, which suggests that abnormal membrane composition may also be involved in PSS.

Correlation analyses showed that there was no linearity between increase in OR and routine haematological indicators. Because the concentration responsible for $50 \%$ haemolysis was abnormally decreased in $75 \%$ of dogs, the OFT could be used as a sensitive indicator for liver metabolic dysfunctions, independent of routinely used haematological tests. Due to the chronic character of metabolic changes occurring in PSS, the OFT could also be introduced in long-term management as an accessory test reserved for patients on medical therapy or after surgical treatment. However, further studies are required, including a series of measurements and correlation analyses with routinely used liver function tests, liver histological changes and liver ultrasound findings.

Haemoglobin concentrations, with $\mathrm{MCH}$ and $\mathrm{MCHC}$ values and changes in OR, were significantly related to the total parenchymal score. More detailed statistical analysis revealed that this relationship, in the case of changes in OR and $\mathrm{MCH}$ values, was due to lipogranuloma formation. The majority of lipogranulomas were with a predominance of lipid vacuoles. The relationship between lipogranuloma formation and changes in OR could indicate an association with lipid metabolism alterations, as lipogranulomas have their origin in focal hepatocyte lipidosis and necrosis (Winkle et al. 2006).

Haemoglobin concentration and MCHC values were also significantly related to the total portal score. However, more detailed analyses did not confirm a relationship between haemoglobin concentrations and lesions of the portal area. MCHC values were dependent on arteriolar proliferation and fibrosis. Fibrosis was however confirmed only in two dogs, the MCHC values of which were within the reference range. The association with arteriolar proliferation remains also undecided.

\section{Acknowledgements}

The authors would like to thank MUDr. Víta Žampachová from the First Department of Pathological Anatomy from the Faculty of Medicine (Masaryk University, Brno, Czech Republic) for her consultations.

\section{References}

Bunch SE, Jordan HL, Sellon RK, Cullen JM, Smith JE 1995: Characterization of iron status in young dogs with portosystemic shunt. Am J Vet Res 56: 853-858

Comazzi S, Paltrinieri S, Spagnolo V, Sartorelli P 2002: Some aspects of erythrocyte metabolism in insulintreated diabetic dogs. Res Vet Sci 72: 23-27

Elias F, Lucas, SRR, Hagiwara MK, Kogika MM, Mirandola RMS 2004: Erythrocyte osmotic fragility in cats with hepatic disease and cats with renal failure. Cięncia Rural 34: 413-418

Isobe K, Matsunaga S, Nakayama H, Uetsuka K 2008: Histopathological characteristics of hepatic lipogranulomas with portosystemic shunt in dogs. J Vet Med Sci 70: 133-138

Lee KCL, Winstanley A, House JV, Lipscomb V, Lamb C, Gregory S, Jalan R, Mookerjee RP, Brockman DJ 2011: Association between hepatic histopathologic lesions and clinical findings in dogs undergoing surgical attenuation of a congenital portosystemic shunt: 38 cases (2000-2004). J Am Vet Med Assoc 239: 638-645

Mathews KG, Bunch SK 2005: Vascular liver diseases. In: Ettinger SJ, Feldman EC: Textbook of veterinary internal medicine. Elsevier, WB Saunders, Philadelphia, pp. 1453-1464

Nakamura M, Takahashi M, Ohno K, Koshino A, Nakashima K, Setoguchi A, Fujino Y, Tsujimoto H 2008 : C-reactive protein concentration in dogs with various diseases. J Vet Med Sci 70: 127-131

Niles JD, Williams JM, Cripps PJ 2001: Hemostatic profiles in 39 dogs with congenital portosystemic shunts. Vet Surg 30: 97-104

Simpson KW, Meyer DJ, Boswood A, White RN, Maskell IE 1997: Iron status and erythrocyte volume in dogs with congenital portosystemic vascular anomalies. J Vet Intern Med 11: 14-19 
Sláma P, Sládek Z, Ryšánek D 2006: Effect of isolation techniques on viability of bovine blood neutrophils. Acta Vet Brno 75: 343-353

Weiss G 2009: Iron metabolism in the anemia of chronic disease. Biochim Biophys Acta 1790: 682-693

Winkle TV, Collen JM, Van den Ingh TSGAM, Charles JA, Desmet VJ 2006: Morphological classification of parenchymal disorders of the canine and feline liver. 3 Hepatic abscesses and granulomas, hepatic metabolic storage disorders and miscellaneous conditions. In: Rothuizen J, Bunch SE, Charles JA et al.: WSAVA Standards for clinical and histological diagnosis of canine and feline liver disease. Elsevier, Oxford, pp. 103-116 